\title{
Maxillofacial Prosthesis with Stages Connected by Magnets for Large Midface Defect: A Case Report
}

\author{
Mohamed Azhari $^{1}$, Hassna Rokhssi ${ }^{2}$, Nadia Merzouk ${ }^{3}$, Oussama Bentahar ${ }^{4}$
}

\begin{abstract}
Prosthetic rehabilitation is done to restore esthetics, function, and improves the quality of life of the patient. Maxillofacial prosthesis is not only a complementary solution to surgery but also a real therapeutic possibility, which helps to restore the defects of large extent. The present case report presented a patient undergone maxillectomy, in which surgery had resulted in loss of intraoral structures, resulting in communication with the nasal fossae and projecting to the facial level by so-called extraoral mutilations. The patient was rehabilitated utilizing maxillofacial stage prosthesis with stages connected with magnets.

Keywords: Magnetic attachments, Maxillary obturator, Maxillofacial prosthesis, Midface defect, Nasal epithesis.

International Journal of Prosthodontics and Restorative Dentistry (2021): 10.5005/jp-journals-10019-1286
\end{abstract}

\section{BACKGROUND}

Maxillofacial prosthesis is utilized for restoring an organ or part of the body that is located at the level of the orofacial region; it may be an endoprosthesis implanted at the level of the tissues or an exoprosthesis in contact with the tissues without any integration. Maxillofacial defects follow surgical resection, trauma, infection, or iatrogenic treatment (bisphosphonates, arsenic, etc.). The maxillary tumors, and especially the carcinomas, are nowadays more frequent and their surgical treatment induces loss of structures in several aspects depending on their seat and volume. The defects are often associated with functional disorders (breathing, phonation, speech, eating, swallowing, chewing), esthetic-related complications that affect the patient psychology and quality of life. ${ }^{1}$

Treatment of the epithelial carcinoma varies depending on the size, depth, and location. Treatment options are surgical removal, chemotherapy, and radiotherapy. Reconstruction must bring a satisfactory restoration of facial esthetics and functions to the patients. Advancement in maxillofacial materials like silicones makes it possible to fabricate obturator prostheses to patients with excellent results. These results are conditioned by a perfect collaboration between the maxillofacial surgeon and the maxillofacial prosthetic dentist. $^{2}$ This paper highlights the rehabilitation of the facial mass after malignant tumor resection utilizing maxillofacial stage prosthesis with stages connected with magnets.

\section{Case Description}

A patient aged 68 years, addicted smoker, operated several times was reported in the department. In 2005, he underwent the surgical exeresis of squamous cell carcinoma at the nasal level. Then, he has been first exposed to excision of the osseous and cartilaginous nose, encroaching on the left cheek area. Afterward, an intraoperative reconstruction with a free frontal flap and a juvenile advancement flap has been exerted. Ten years later, this patient has secondly taken in charge for a recurrence of the lesion with invasion of the palatal blade, which resulted in an excision of almost the entire maxilla. The defect was surgically reconstructed by an advancement and labial flap. The surgical therapeutic was combined with the chemotherapy and radiotherapy. During the visual test, many
${ }^{1-4}$ Department of Prosthodontics, Faculty of Dentistry, University Mohamed V, Rabat, Morocco

Corresponding Author: Mohamed Azhari, Department of Prosthodontics, Faculty of Dentistry, University Mohamed V, Rabat, Morocco, Phone: +212661691307, e-mail: azhari_med@yahoo.fr

How to cite this article: Azhari M, Rokhssi H, Merzouk $N$, et al. Maxillofacial Prosthesis with Stages Connected by Magnets for Large Midface Defect: A Case Report. Int J Prosthodont Restor Dent 2021;11(1):61-65.

Source of support: Maxillofacial Prosthodontics Unit - Center of Consultation and Dental Treatment of RABAT- Morocco

Conflict of interest: None

scars were noticed due to the shreds of reconstruction and total amputation of the extended nasal pyramid on the left cheek (Figs 1A and B). The lower floor was symmetric and without any anomalies. There was no pain while palpation; however, a slight intraorbital hypoesthesia was noticed. The cervical examination showed no cervical lymphadenopathies and no palpable mass. The endobuccal examination showed completely edentulous maxillary defect with an oronasal communication of $1 \mathrm{~cm}$ in diameter.

The prosthetic challenges faced during the rehabilitation were due to squeal of oncologic therapy, combined intra- and extraoral defect, the limited mouth opening, xerostomia, scar bridles (reconstruction flaps), complete absence of maxillary and mandibular dentition, and psychological component (facial disfigurement). These problems can be classified into biological, functional, esthetic, and psychological issues. For this case, the surgical reconstruction option was difficult due to volume of defect and the psychological state of the patient who was operated several times. Therefore, it was planned to provide maxillofacial prostheses to the patient. Rehabilitation of maxillofacial defects had been done in three prosthetic segments connected together by magnetic attachments: Lower segment: consisting of a complete removable dental prosthesis restoring absent dentition and surrounding structures; average segment: represented by an intermediate piece restoring the oronasal communication; and upper segment: allows the restoration of the extraoral defect. 


\section{First Prosthetic Stage: Intermediate Piece (Average Segment)}

The impression of oronasal communication was made using a high-viscosity silicone impression material in an extraoral way.
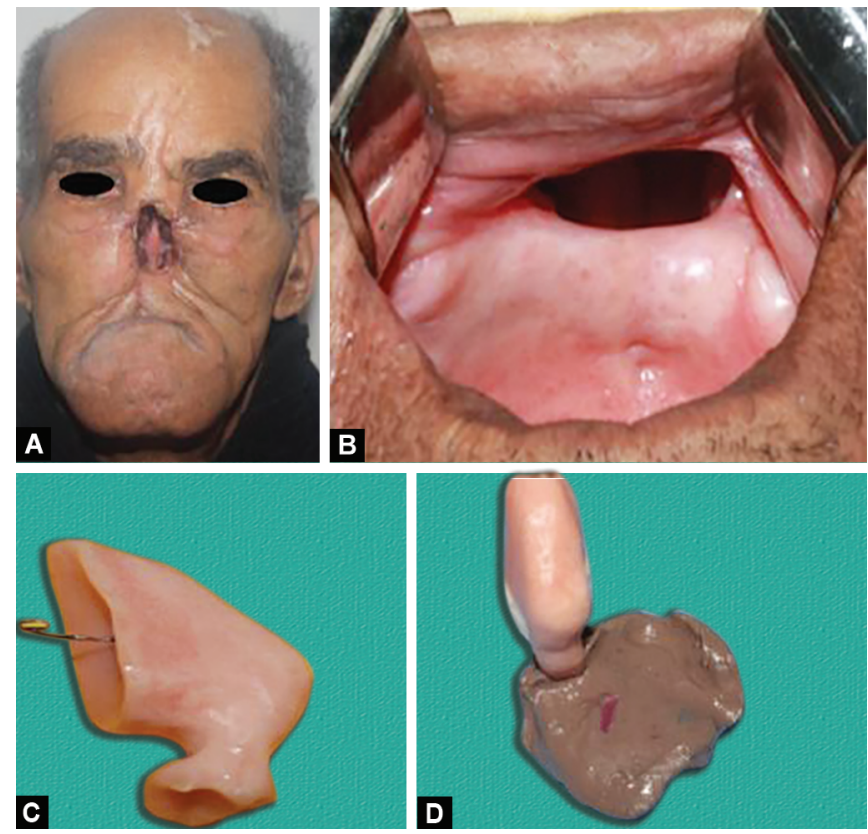

Figs 1 A to D: (A) Extraoral view; (B) Intraoral view; (C) Intermediate piece; (D) Maxillary impression with intermediate piece
This molding has transformed into a wax model that was used to fabricate the intermediate piece with heat-cured acrylic resin (lost wax technique). This prosthetic stage was tried and adapted to the defect (Fig. 1C).

\section{Second Prosthetic Stage: Dental Prosthesis (Lower Segment)}

The intraoral impression was made in two steps. The first step was represented as anatomical impression made with alginate, to obtain a primer model on which another impression was build up to make functional impression with polyether material for the peripheral joint and the polysulfide regular material for the surfacing. This impression had been also extended to record the position of the intraoral segment of the intermediate part (Fig. 1D).

The maxillomandibular relation has been recorded using classical techniques for complete removable prosthesis utilizing the Ballard class III promandibulie scheme. Taking into account the quality of the fragile maxillary support surface and to reinforce the prosthetic balance, the classic triad of Housset ${ }^{3}$ was applied to provide the tightness for the therapeutic success. The position of the occlusion plane was very important for this equilibrium, which brings it closer to the possible limit of the maxillary support surface and also influences the esthetics of the patient. Centric relation with an undervalued vertical dimension was maintained to avoid any trauma to the bearing surface with more possibility for maxillofacial physiotherapy by minimizing prosthetic space. The teeth arrangement was done in a cross-functional assembly (Figs 2A and B) to provide better orientation of occlusal forces in levitation polygons to provide prosthetic balance in static and
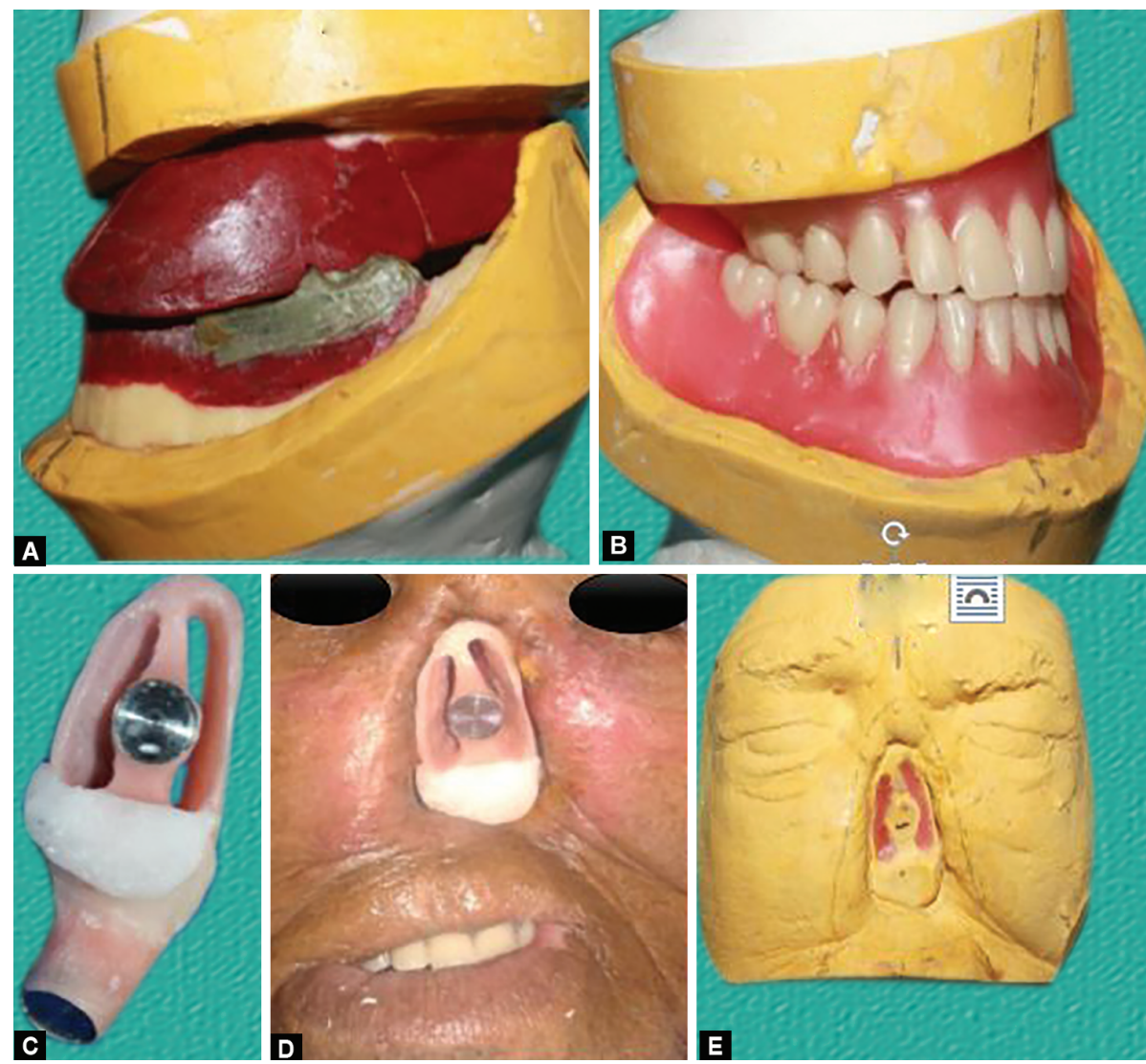

Figs 2A to E: (A) Recording maxillomandibular relation; (B) Prosthetic teeth assembly; (C) Fixing magnets on intermediate piece; (D) Checking adaptation of dental prosthesis and intermediate piece after magnetic connection; (E) Facial mold 

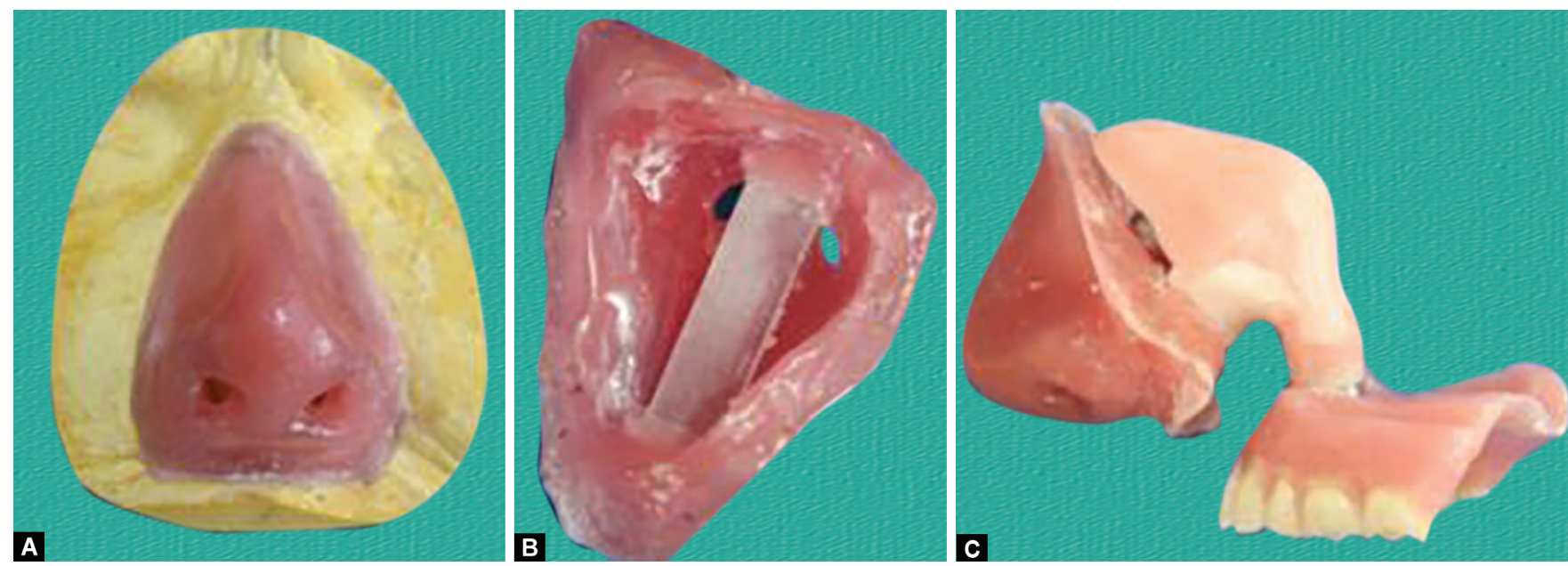

Figs 3A to C: (A) Nasal wax up; (B) Insertion of resin plate; (C) Fixing magnets on the resin plate and checking the peripheral seal

dynamic occlusion. Provision for the full balance during extrusive movements and avoidance of cheek bite by cusp cover (inverted occlusion).

\section{Third Prosthetic Stage: Nasal Prosthesis (Upper Segment)}

The facial impression was made after positioning the intermediate piece above the dental prosthesis. The facial impression was made by the partial molding, a technique described by Pomar and Soulet. ${ }^{4}$ The alginate impression was supported by a plaster screed; this impression was also used to record the position of the extraoral end of the intermediate piece (Figs $2 C$ and D). The impression of the nasal epithesis was made on a donor preferably from the same family with a certain resemblance to the patient. This impression was made by the alginate impression material with a classical partial facial impression technique. After treatment and wax casting of nasal impression, a nasal wax model (Fig. 2E) was obtained. An artistic integration of this model on master facial mold was done. Next, a resin plate, which served as a support for counterpart of magnetic attachment, was fixed at the lower surface of the waxed-up make-up model (Fig. 3A). On the patient, the model was tried, checking that the resin plate does not interfere with the edges of nasal defect and that peripheral seal to be hermetic (Fig. 3B). After that, the magnets were fixed on the resin plate on the one hand and on the upper surface of the intermediate piece on the other hand (Fig. 3C). After validation of the wax model, it was invested in silicone using the plaster mold technique. For this, a transparent high-temperature vulcanizing silicone, colored in mass with pigments according to patients' skin color, was used. Indeed, three pigments were generally used at this stage: titanium white for opacity, cobalt blue for the venous component, and vermilion red for the arterial component. In the prosthetic insertion phase, the color of the epithesis was integrated with that of the tegument via a peripheral pigmentation by the use of certain pigments such as natural sienna, ocher yellow, dead head, etc.

The final phase consists of fixing magnetic attachments (Multipurpose magnet MPMS-H2.5D9.4/Technovent 41TEMPMS) using transparent resin drops and respecting predefined position during the impression stage, the axis of insertion of the prosthetic segments, and perfect adaptations of the prosthesis during function without play or mobility (Fig. 4).
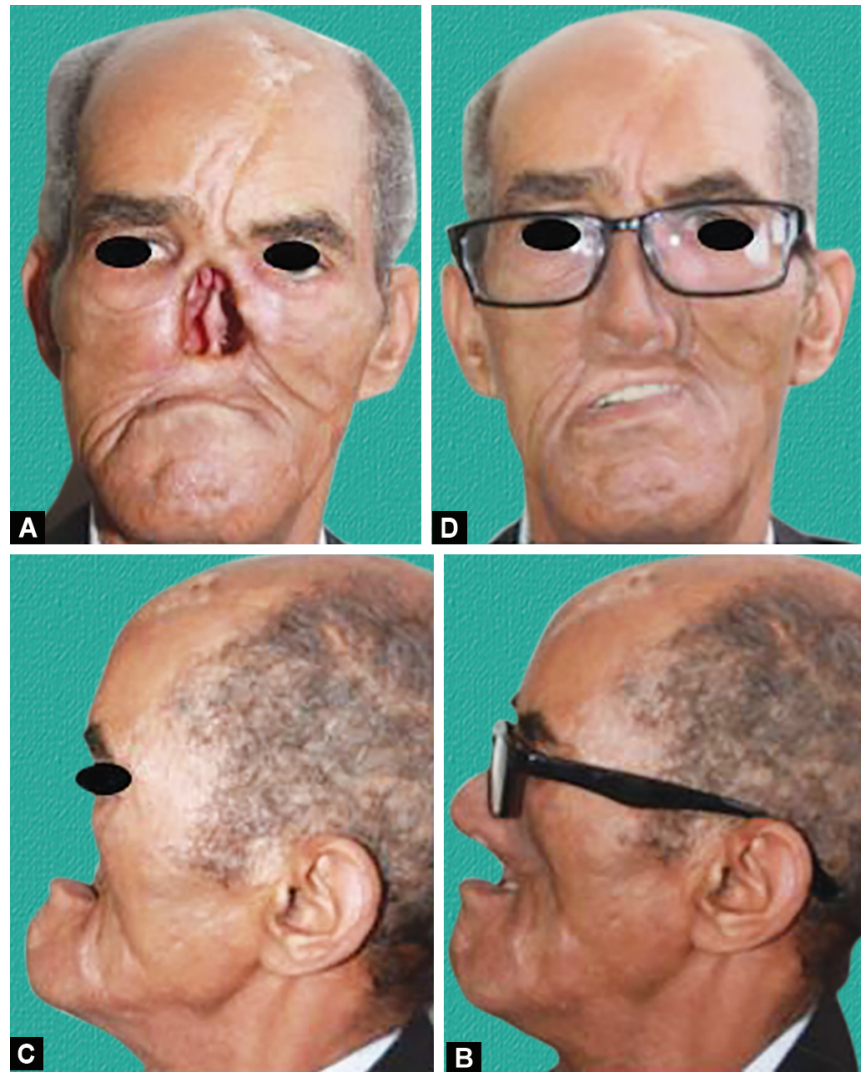

Figs 4A to D: Final result after manufacturing of nasal epithesis

\section{Discussion}

After the surgery for tumor excision of the orofacial region, the patient generally suffers from an amputation and socioprofessional isolation, with a consequent impairment of his quality of life. Surgical reconstruction solution with advent of flap and implantable biomaterial procedures seems the treatment of choice. However, some contraindications of surgery, either local or general, may recommend prosthetic rehabilitation as a treatment option, which itself benefits from advances in biomaterials, implants, and digital tools. 
Clinical examination should collect enough clinical data, which may be useful for prosthetic restoration. The patient presented in this case report was operated on several occasions, with failures, relapses, and therapeutic complications. The patient therefore presents a much-deteriorated state of physical and mental health. In fact, prosthetic management, although constitutes first-aid therapy for the said patient, is however complex given the problematic context in the field.

The success of any type of prosthetic rehabilitation of these defects requires close coordination between the surgeon and the prosthodontist. Thus, for example, the conservation during tumor excision surgery of undercuts and sufficient bone supports (alveolar crest, nasal spine, palatal edge) is of great use for prosthetic balance. $^{5}$

Prosthetic balance is one of the complex challenges for the practitioner. In fact, the retention of these devices always involves the judicious exploitation of the support structures by the use of adapted individual impression trays and respecting the play of adjacent mobile tissues by anatomofunctional impressions. Polished stabilizing surfaces are also of great interest for these devices; thus, we recommend use of tertiary piezographic impressions. ${ }^{6}$

Recording of the intermaxillary report is of paramount importance. Indeed, for the patient a reference relation position centered was opted since temporomandibular joints are intact, and vertical dimension was slightly underestimated for better masticatory efficiency. Balanced occlusion was given, which was very essential for the prosthetic balance.

In the present case, the defect involves oral and nasal cavities and externalizing by a loss of the nasal organ, causing facial disfigurement. Therefore, prosthetic management was done by the restoration of the oral cavity with a maxillary obturator prosthesis and extraoral defect by a nasal epithesis. The biomechanical problem encountered was twofold, since two prosthetic components having destabilizing forces in different directions have to be considered. Thus, the intermediate piece makes it possible to create a system of balance and cancellation of the stall forces. ${ }^{8}$ Position and the orientation of magnets is of great importance for prosthetic balance. Indeed, attractive forces of magnets parallel to each other enhance the prosthetic retention, by avoiding destabilizing oblique components. ${ }^{9}$ Retention was further improved extraorally by the use of glasses frame, which ensured keeping in place the epithesis and promoted prosthetic balance. ${ }^{10}$

In the present clinical situation, the biomechanical management of retention by attachment was adopted from Bidra et al. ${ }^{11}$ Magnetic retention is not sufficient considering importance of volume of defect and tissue retraction, so it is advised to use maxillary implants for improving prosthetic balance. In the present case, the retention was found to be favorable since the support surface was utilized and the advantage of the form of the reduced extraoral and intraoral communication was taken for perfect adaptation of the intermediate piece that was considered as a pillar of success in present restoration. Another situation that ruled out the implant in the present case was the number of surgical interventions and the significant dose of radiotherapy that was received by the patient.

Resin was used to fabricate intermediate obturator in the present case. Generally silicones can be used as a flexible material as reported by Dholam et al. ${ }^{12}$ to exploit the zones of withdrawal as much as possible. In the present case, silicone was not used as the handling of silicone was difficult both in terms of production and in terms of means of attachments. Silicone also has significant biodegradability of the part, which is highly exposed to oral and nasal fluids.

Prosthetic rehabilitation must take into account the hypooxygenated and hypovascularized state of tissues following anticancer therapy. Indeed, any trauma to the weakened support surface can be complicated by ulceration, osteoradionecrosis, or even with recurrence of the tumor lesion. This is why the prosthesis must be adapted to the support surface as much as possible without overextension or compression. To meet these specifications, nontraumatic impression techniques and materials were opted, with the use of polyether and polysulfide over hydrophilic zinc oxide, which is contraindicated in the presence of xerostomia. The vertical dimension must be judiciously underestimated to minimize the occlusal constraints. Maxillofacial kinestherapy was started to improve the context of microstomia generated by the surgical flap technique. $^{13}$

Prosthetic rehabilitation was not alone a solution for the problems of these defects. Indeed collaboration with the speech therapist was essential, so a phonetic reduction was prescribed during and after prosthetic realization to correct phonetic disorders. The practitioner must take the problem of chewing and swallowing into account by adopting a balanced occlusal concept and a maxillomandibular relation, which contributes to masticatory efficiency. ${ }^{14}$

In extraoral prosthetic restoration, the main objective was the dissipation of the facial disfiguration by a nasal epithesis while simulating in an artisanal way shape, color, and dimension of the lost organ. For this, the old photographs taken before tumor attack are important and also the use of an impression of a donor subject as done in present clinical situation to facilitate the artistic work by the molding technique. The peripheral joint of epithesis constitutes major problem for extraoral restoration. Learning skin prosthesis limit, which is detrimental from an esthetic point of view, and peripheral make-up or the use of glasses frames or moustaches to ensure camouflage should be followed. ${ }^{15}$

In intraoral prosthesis restoration, respecting the esthetic parameters in conventional complete removable prosthesis, in particular good lip and cheek support, a good situation and orientation of the occlusion plane, a well-estimated vertical dimension, and a choice respecting esthetics rules of prosthetic teeth, is very essential. ${ }^{16}$

Patients presenting with defects at the level of the orofacial region generally have difficulties to accept the lesion and considered as a true handicap and it isolates them socially. The care of such patients should not devoted to the restoration of the lost organ only but so far to improve self-confidence through behavioral or drug psychotherapy. ${ }^{17}$

Maxillary obturator prosthesis for midface defect is extremely important for restoration of mastication, speech, swallowing, respiration, esthetic, and consequently to improve a quality of life of these patients. That is why, rehabilitation of these defects must be done on a close symbiotic cooperation between the surgeon and the prosthodontist. ${ }^{18}$ Maxillofacial rehabilitation can now benefit from the advent of digital design and manufacturing tools. However, contribution of the artistic touch is still essential, although the quality of the numeric design, in fact at this time we have not yet 3D printers working with silicones, the printing carried out in resin generally and therefore a passage by a molding step is essential. ${ }^{19}$ 


\section{References}

1. Ackerman AJ. Maxillofacial prosthesis. Oral Surg Oral Med Oral Pathol 1953;6(1):176-200. DOI: 10.1016/0030-4220(53)90152-2.

2. de Caxias FP, dos Santos DM, Bannwart LC, et al. Classification, history, and future prospects of maxillofacial prosthesis. Int J Dent 2019. 8657619. DOI: 10.1155/2019/8657619.

3. Housset $P$. Sustentation, stabilisation, rétention: triade d'équilibre. Prat Odonto Stomatol 1957;495:1-9.

4. Pomar P, Soulet $H$. Empreinte fragmentée: contribution à la réhabilitation prothétique de l'édenté total après Maxillectomie. Actual Odontostomatol Encycl Prat 1995(191):443-447.

5. Phasuk K, Haug SP. Maxillofacial prosthetics. Oral Maxillofac Surg Clin North Am 2018;30(4):487-497. DOI: 10.1016/j.coms.2018.06.009.

6. Beumer IIIJ, Marunick MT, Esposito SJ. Maxillofacial rehabilitation surgical and prosthodontics management of cancer-related acquired, and congenital defects of the head and neck. 3rd ed., 2011.

7. Hüe $\mathrm{O}$, Berteretche MV. Prothèse complète: Réalité clinique-Solutions thérapeutiques. Paris: Quintessence International; 2004. p 289.

8. Supassra N, Prana S, Natdhanai C, et al. Combination prosthetic design providing a superior retention for mid-facial defect rehabilitation: a case report. J Clin Exp Dent 2017;9(4):e590-e594.

9. Drago CJ. Tarnish and corrosion with the use of intraoral magnets. J Prosthet Dent 1991;66(4):536-540. DOI: 10.1016/0022-3913(91) 90519-3.

10. Guttal SS, Alva B, Nadiger RK. Use of a stud attachment to retain a silicone orbital prosthesis: a clinical report. J Prosthodont 2012;21(4):317-321. DOI: 10.1111/j.1532-849X.2011.00812.x.

11. Bidra AS, Montgomery PC, Jacob RF. Maxillofacial rehabilitation of a microstomic patient after resection of nose, lip, and maxilla.
J Oral Maxillofac Surg 2010;68(10):2513-2519. DOI: 10.1016/j. joms.2010.05.046.

12. Dholam KP, Sadashiva KM, Bhirangi PP. Rehabilitation of large maxillary defect with two-piece maxillary obturator. J Cancer Res Ther 2015;11(3):664. DOI: 10.4103/0973-1482.140801.

13. Psaume-Vandebeek D. Kinesiotherapy in maxillofacial practice. Actual Odontostomatol (Paris) 1991;45(174):171-190.

14. dos Santos DM, de Caxias FP, Bitencourt SB, et al. Oral rehabilitation of patients after maxillectomy. A systematic review. J Oral Maxillofac Surg 2018;56(4):256-266. DOI: 10.1016/j.bjoms.2018. 03.001

15. Becker C, Becker AM, Dahlem KKK, et al. Aesthetic and functional outcomes in patients with a nasal prosthesis. Int J Oral Maxillofac Surg 2017;46(11):1446-1450. DOI: 10.1016/j.ijom.2017. 04.024 .

16. Gastaldi G, Palumbo L, Moreschi C, et al. Prosthetic management of patients with oro-maxillo-facial defects: a long-term follow-up retrospective study. Oral Implantol (Rome) 2017;10(3):276-282. DOI: 10.11138/orl/2017.10.3.276.

17. Breeze J, Rennie A, Morrison A, et al. Health-related quality of life after maxillectomy: obturator rehabilitation compared with flap reconstruction. Br J Oral Maxillofac Surg 2016;54(8):857-862. DOI: 10.1016/j.bjoms.2016.05.024.

18. Mathar MI, Shamsudeen SM. Maxillofacial rehabilitation of nasal defect with nasal prosthesis using donor method: a case report. Nigerien J Clinl Pract 2020;23(7):1022-1025. DOI: 10.4103/njcp. njcp_657_19.

19. Pan S, Liu B. Research progress on 3D printing metal powders used in cranio-maxillofacial prosthesis. Hua Xi Kou Qiang Yi Xue Za Zhi 2019;37(4):438-442. 